\title{
Production-process modelling based on production-management data: a Petri-net approach
}

\author{
D. GRADIŠAR* $+\dagger$ and G. MUŠIČ $\dagger$ \\ $\dagger$ Faculty of Electrical Engineering, University of Ljubljana, Tržaška 25, 1000 Ljubljana, Slovenia \\ $\$$ Jozef Stefan Institute, Jamova 39, 1000 Ljubljana, Slovenia
}

\begin{abstract}
During the development of a production control system, an appropriate model of the production process is needed to evaluate the various control strategies. This paper describes how to apply timed Petri nets and existing production data to the modelling of production systems. Information concerning the structure of a production facility and the products that can be produced is usually given in production-data management systems. We describe a method for using these data to construct a Petri-net model algorithmically. The timed Petri-net simulator, which was constructed in Matlab, is also described. This simulator makes it possible to introduce heuristics, and, in this way, various production scenarios can be evaluated. To demonstrate the applicability of our approach, we applied it to a scheduling problem in the production of furniture fittings.
\end{abstract}

Keywords: Timed Petri nets; Modelling; Scheduling; Production systems; Simulation

\section{Introduction}

As the role played by information systems in production control increases, the need for a proper evaluation of the various decisions in both the design and operational stages of such systems is becoming more and more important.

In general, an appropriate model of a production process is needed in order to cope with its behaviour. However, this behaviour is often extremely complex. When the behaviour is described by a mathematical model, formal methods can be used, which usually improve the understanding of systems, allow their analysis and help in implementation. Within the changing production environment the effectiveness of production modelling is, therefore, a prerequisite for the effective design and operation of manufacturing systems.

Scheduling is a fundamental problem in the control of any resource-sharing organization. Scheduling problems are very complex and many have been proven to be NP hard (Jain and Meeran 1999). There are several major approaches to scheduling. Formal, theoretically oriented approaches have to ignore many practical constraints in order to solve these problems efficiently (Richard and
Proust 1998). This is the reason why only a few real applications exist in the industrial environment (Hauptman and Jovan 2004). Mathematical programming approaches are computationally demanding and often cannot achieve feasible solutions to practical problems (Jain and Meeran 1999). Soft-computing approaches, e.g. genetic algorithms and neural networks, require considerable computation and only yield sub-optimal solutions. Instead, heuristic dispatching rules (Panwalker and Iskaneder 1977, Blackstone et al. 1982), such as Shortest Processing Time (SPT) or Longest Processing Time (LPT), are commonly used in practice. An interesting property of heuristic dispatching rules is that they can easily be used in conjunction with production models derived within different mathematical modelling frameworks, e.g. the disjunctive graph model (Błażewicz et al. 1996, 2000), timed automata (Abdeddaim et al. 2006), and Petri nets (Murata 1989, Zhou and Venkatesh 1999).

Petri nets (PN) represent a powerful graphical and mathematical modelling tool. The different abstraction levels of Petri-net models and their different interpretations make them especially usable for life-cycle design (Silva and Teruel 1997). Many different extensions of classical Petri

*Corresponding author. Email: dejan.gradisar@ijs.si

International Journal of Computer Integrated Manufacturing 
nets exist, and these are able to model a variety of real systems. In particular, timed Petri nets can be used to model and analyse a wide range of concurrent discreteevent systems (Zuberek 1991, Van der Aalst 1996, Zuberek and Kubiak 1999, Bowden 2000). Several previous studies have addressed the timed-Petri-net-based analysis of discrete-event systems. López-Mellado (2002), for example, deals with the simulation of the deterministic timed Petri net for both timed places and timed transitions by using the firing-duration concept of time implementation. Van der Aalst (1998) discusses the use of Petri nets in the context of workflow management. Gu and Bahri (2002) discuss the usage of Petri nets in the design and operation of a batch process. There is a lot of literature on the applicability of PNs in the modelling, analysis, synthesis and implementation of systems in the manufacturing-applications domain. A survey of the research area and a comprehensive bibliography can be found in Zhou and Venkatesh (1999). Recalde et al. (2004) give an example-driven tour of Petri nets and manufacturing systems where the use of Petri-net production models through several phases of the design life-cycle is presented.

A straightforward way of using the heuristic rules within a Petri-net modelling framework is to incorporate the rules for the conflict-resolution mechanism in an appropriate Petri-net simulator. Many different Petri-net simulators exist, some of which also support timed Petri nets, and they usually support random decisions to make a choice in the case of conflicts. The Petri-net toolbox for Matlab (Matcovschi et al. 2003) allows the use of priorities or probabilities to make a choice about a conflicting transition to fire. CPN Tools can also be used for the modelling, simulating and analyses of untimed and timed Petri nets (Ratzer et al. 2003).

One of the central issues when using Petri nets in manufacturing is the systematic synthesis of Petri-net models for automated manufacturing systems. Problems arise when the complexity of a real-world system leads to a large Petri net having many places and transitions (Zhou et al. 1992). A common approach is to model the components and build the overall systems in a bottom-up manner. However, a Petri net constructed by merging arbitrary sub-nets is difficult to analyse, and, furthermore, an early design error can lead to an incorrect model. Zhou et al. (1992) propose a hybrid methodology that builds a model by combining the top-down refinement of operations and the bottom-up assignment of resources. Another approach is the use of well-defined net modules and restricting their interaction. By merging corresponding sub-nets in a predefined way a set of desired properties of the resulting net is maintained (Jeng 1997). But the synthesis of complex models remains tedious and errorprone. Therefore, a number of researchers have put toward the idea of modelling a flexible manufacturing system
(FMS) with a FMS modelling language. The language model is then automatically translated into one of the standard PN classes, such as Coloured Petri nets - CPN (Arjona-Suarez and Lopez-Mellado 1996) or Generalized stochastic Petri nets - GSPN (Xue et al. 1998). Some researchers have also proposed the translation into special PN classes, e.g. B-nets ( $\mathrm{Yu}$ et al. 2003a). Other approaches to the automatic synthesis of PN models are presented by Camurri et al. (1993), Ezpeleta and Colom (1997) and Basile et al. (2006) and special PN classes appropriate for modelling FMS appear in Proth et al. (1997), Van der Aalst (1998) and Janneck and Esser (2002).

On the other hand, Huang et al. (1995) use a discreteevent matrix model of a FMS, which can be built based on standard manufacturing data, and can also be interpreted as a Petri net. This latter approach is particularly attractive when there are data concerning the production process available within some kind of production-management information system. Using these data, a model-building algorithm can be embedded within the information system. In this paper we propose a method for using the data from management systems, such as Manufacturing Resource Planning (MRP II) systems (Wortmann 1995), to automate the procedure of building up the Petri-net model of a production system. Instead of using a discrete-event matrix model, the Petri net is built directly in a top-down manner, starting from the bill of materials (BOM) and the routings (Wortmann 1995). The BOM and the routing data, together with the available resources, form the basic elements of the manufacturing process. These data can be effectively used to build up a detailed model of the production system with Petri nets. The product structure given in the form of the BOM and the process structure in the form of routings have also been used by other researchers. Czerwinski and Luh (1994) propose a method for scheduling products that are related through the BOM using an improved Lagrangian Relaxation technique. An approach presented by Yeh (1997) maintains production data by using a bill of manufacture (BOMfr), which integrates the BOM and the routing data. Production data are then used to determine the production jobs that need to be completed in order to meet demands. Compared to previous work, the method proposed in this paper builds a Petri-net model that can be further analysed, simulated and used for scheduling purposes.

First we define timed Petri nets, where time is introduced by using the holding-durations concept. A general class of place/transition $(\mathrm{P} / \mathrm{T})$ nets supplemented by timed transitions is used. Although several special classes of PNs have been defined, there was no need to either restrict the behaviour of the $\mathrm{P} / \mathrm{T}$ nets or extend their modelling power during the work presented in this paper. The use of some kind of high-level Petri nets would, however, probably be needed in a real industrial implementation. Practical 
experience also shows that, for most applications in a real manufacturing environment, the use of deterministic time delays is sufficient. Adopting the class of timed $\mathrm{P} / \mathrm{T}$ nets, a method for modelling the basic production activities with such a Petri net is described. A corresponding algorithm of automatic model building is presented. For a defined, timed Petri net a simulator was built, for which different heuristic rules can be introduced for scheduling purposes. The applicability of the proposed approach was illustrated using a practical scheduling problem, where data concerning the production facility is given with the BOM and the routings. The model constructed using the proposed method was used to determine a schedule for the production operations.

In the next section we describe timed Petri nets that can be used for the modelling and analysis of a production system. In section 3 the method for modelling the production system using data from the production-management system is presented. Section 4 explains the simulator/scheduler that was built for the purposes of scheduling; it can use different heuristic dispatching rules. An illustrative application of modelling an assembly process and developing a schedule using timed Petri nets is given in section 5. Finally, the conclusions are presented in section 6 .

\section{Timed Petri nets}

Petri nets are a graphical and mathematical modelling tool that can be used to study systems that are characterized as being concurrent and asynchronous.

The basic Place/Transition Petri net (Zhou and Venkatesh 1999) is represented by the multiple

$$
P N=\left(P, T, I, O, M_{0}\right),
$$

where $P=\left\{p_{1}, p_{2}, \ldots, p_{g}\right\}$ is a finite set of places; $T=t_{1}$, $t_{2}, \ldots, t_{h}$ is a finite set of transitions; $I:(P \times T) \rightarrow \mathbb{N}$ is the input arc function. If there exists an arc with weight $k$ connecting $p_{i}$ to $t_{j}$, then $I\left(p_{i}, t_{j}\right)=k$, otherwise $I\left(p_{i}, t_{j}\right)=0$; $O:(P \times T) \rightarrow \mathbb{N}$ is the output arc function. If there exists an arc with weight $k$ connecting $t_{j}$ to $p_{i}$, then $O\left(p_{i}, t_{j}\right)=k$, otherwise $O\left(p_{i}, t_{j}\right)=0 ; M: P \rightarrow \mathbb{N}$ is the marking; and $M_{0}$ is the initial marking.

Functions $I$ and $O$ define the weights of the directed arcs, which are represented by numbers placed along the arcs. In the case when the weight is 1 , this marking is omitted, and in the case when the weight is 0 , the arc is omitted. Let $\bullet t_{j} \subseteq P$ denote the set of places which are inputs to transition $t_{j} \in T$, i.e. there exists an arc from every $p_{i} \in \bullet t_{j}$ to $t_{j}$. A transition $t_{j}$ is enabled by a given marking if, and only if, $M\left(p_{i}\right) \geq I$ $\left(p_{i}, t_{j}\right), \forall p_{i} \in \bullet t_{j}$. An enabled transition can fire, and as a result remove tokens from input places and create tokens in output places. If the transition $t_{j}$ fires, then the new marking is given by $M^{\prime}\left(p_{i}\right)=M\left(p_{i}\right)+O\left(p_{i}, t_{j}\right)-I\left(p_{i}, t_{j}\right), \forall p_{i} \in P$.
The structure of the Petri net can also be given in a matrix representation (Zhou and Venkatesh 1999). We define a $g \times h$ input matrix $\mathbf{I}$, whose $(i, j)$ entry is $I\left(p_{i}, t_{j}\right)$. Similarly, we define an output matrix $\mathbf{O}$ of the same size, whose elements are defined by $O\left(p_{i}, t_{j}\right)$. Matrices I and $\mathbf{O}$ precisely describe the structure of the Petri net and make it possible to explore the structure using linear algebraic techniques. Furthermore, the marking vector $\mathbf{M}$ where $M_{i}=M\left(p_{i}\right)$, and a firing vector $\mathbf{u}$ with a single nonzero entry $u_{j}=1$, which indicates a transition $t_{j}$ that fires, are defined. Using these matrices we can now write a state equation $\mathbf{M}_{k+1}=\mathbf{M}_{k}+(\mathbf{O}-\mathbf{I}) \cdot \mathbf{u}_{\mathbf{k}}$. The subscript $k$ denotes the $k$ th firing in some firing sequence.

An important concept in PNs is that of conflict. Two events are in conflict if either one of them can occur, but not both of them. Conflict occurs between transitions that are enabled by the same marking, where the firing of one transition disables the other transition. Also, parallel activities or concurrency can easily be expressed in terms of a PN. Two events are parallel if both events can occur in any order without conflicts. A situation where conflict and concurrency are mixed is called a confusion.

The concept of time is not explicitly given in the original definition of Petri nets. However, for the performance evaluation and scheduling problems of dynamic systems it is necessary to introduce time delays. Given that a transition represents an event, it is natural that time delays should be associated with transitions. Time delays may be either deterministic or stochastic. In this work, timed Petri nets with deterministic time delays are used to model the behaviour of a production system.

As described by Bowden (2000) there are three basic ways of representing time in Petri nets: firing durations, holding durations and enabling durations. The firingduration principle says that when a transition becomes enabled it removes the tokens from input places immediately but does not create output tokens until the firing duration has elapsed. Zuberek (1991) gives a well-defined description of this principle. When using the holdingduration principle, a created token is considered unavailable for the time assigned to the transition that created the token. The unavailable token cannot enable a transition and therefore causes a delay in the subsequent transition firings. This principle is graphically represented in figure 1, where the available tokens are schematized with the corresponding number of undistinguishable (black) tokens and the unavailable tokens are indicated by the center not being filled. The time duration of each transition is given beside the transition, e.g. $f\left(t_{1}\right)=t_{\mathrm{d}}$. When the time duration is 0 this denotation is omitted. In figure $1, t$ denotes a model time represented by a global clock and $t_{\mathrm{f}}$ denotes the firing time of a transition. With enabling durations the firing of the transitions happens immediately and the time delays are 

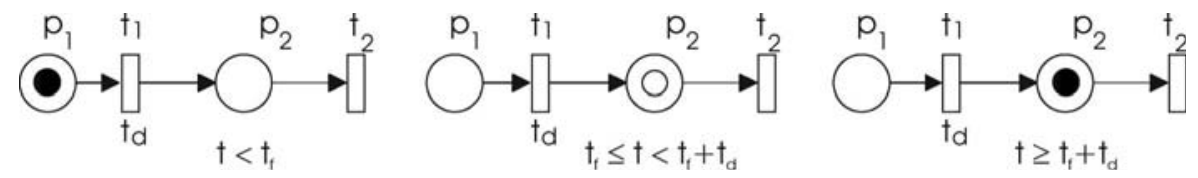

Figure 1. Timed Petri net with holding durations.

represented by forcing transitions that are enabled to stay so for a specified period of time before they can fire.

Holding durations and firing durations are in fact the same way of representing time. We prefer the use of holding durations, because in comparison with firing durations they do not have transitions that remain active over periods of time. Thus, the schematics of holding durations are closer to those of non-timed Petri nets. The main difference between using holding and enabling durations can be seen in a Petri net where confusion appears. In this case, more transitions are enabled by one marking. When the enabling duration policy is used, the firing of one transition can interrupt the enabling of other transitions, as the marking, which has enabled the previous situation, has changed (Bowden 2000). It is reasonable to use holding durations when modelling production processes where the operations are non pre-emptive.

By using holding durations the formal representation of the timed Petri net is extended with the information of time, represented by the multiple

$$
T P N=\left(P, T, I, O, s_{0}, f\right)
$$

where $P, T, I, O$ are the same as above, $s_{0}$ is the initial state of the timed Petri net, and $f: T \rightarrow I R_{0}^{+}$is the function that assigns a non-negative deterministic time-delay to every $t_{j} \in T$. The delays can be represented by $1 \times \mathrm{h}$ row vector $\mathbf{f}$ whose $j$ th entry is $f\left(t_{j}\right)$.

The state of a timed Petri net is a combination of three functions

$$
s=(m, n, r),
$$

where $m: P \rightarrow \mathbb{N}$ is a marking function of available tokens. It defines a $g \times 1$ column vector $\mathbf{m}$ whose $i$ th entry is $m\left(p_{i}\right)$; $n: P \rightarrow \mathbb{N}$ is a marking function of unavailable tokens. It defines a $g \times 1$ column vector $\mathbf{n}$ whose $i$ th entry is $n\left(p_{\mathrm{i}}\right)$; and $r$ is the remaining-holding-time function that assigns values to a number of local clocks that measure the remaining time for each unavailable token (if any) in a place. Assuming $l$ unavailable tokens in $p_{i}$, i.e. $n\left(p_{i}\right)=l$, the remaining-holding-time function $r\left(p_{i}\right)$ defines a vector of $l$ positive real numbers denoted by $\mathbf{r}\left(p_{i}\right)=\left[r\left(p_{i}\right)[1]\right.$, $r\left(p_{i}\right)[2], \ldots, r\left(p_{i}\right)[l] ; ; r$ is empty for every $p_{i}$, where $n\left(p_{i}\right)=0$.

A transition $t_{j}$ is enabled by a given marking if, and only if, $m\left(p_{i}\right) \geq I\left(p_{i}, t_{j}\right), \forall p_{i} \in \bullet t_{j}$. The firing of transitions is considered to be instantaneous. A new local clock is created for every newly created token and the initial value of the clock is determined by the delay of the transition that created the token. When no transition is enabled, the time of the global clock is incremented by the value of the smallest local clock. An unavailable token in a place where a local clock reaches zero becomes available and the clock is destroyed. The enabling condition is checked again. The procedure for determining a new state is described in detail in section 4 .

\section{The modelling of production systems}

This section deals with models for production facilities. These models play a role in the design and the operational control of a plant. Petri nets are a family of tools that provide a framework or working paradigm which can be used for many of the problems that appear during the lifecycle of a production system (Silva and Teruel 1997). If used in all stages, an additional benefit of improving the communication between these stages is achieved.

We present a method for modelling production systems using timed Petri nets based on data from productionmanagement systems for the purpose of performance control. Van der Aalst (1996) provides a method for mapping scheduling problems onto timed Petri nets, where the standard Petri-net theory can be used. To support the modelling of scheduling problems, he proposed a method to map tasks, resources and constraints onto a timed Petri net. In this paper a different representation of time in Petri nets is used, and the structure of the model is derived from existing production-management data (Wortmann 1995). A method for recognizing basic production elements from the management system's database is provided.

When timed Petri nets are used, it is possible to derive performance measures such as makespan, throughput, production rates, and other temporal quantities. In this work the simulation of a timed Petri net is used to estimate the performance measures and evaluate different priority rules.

\subsection{The class of production system}

With the method presented here, several scheduling problems that appear in production systems can be solved. The production systems are considered where management systems (MRP II) are used to plan the production process. 
The system generates work orders that interfere with the demands for the desired products. Different jobs are needed to produce a desired product. In general, more operations have to be performed using different resources in order to complete a specific job. To complete a specific product, more sub-products may be needed. The BOM defines a list of components. These components determine sub-jobs that are needed to manufacture a parent item. In this way the general scheduling problem is defined and can be given as:

- $n$ jobs are to be processed: $J=\left\{J_{j}\right\}, j=1, \ldots, n$;

- $m$ resources are available: $M=\left\{M_{i}\right\}, i=1, \ldots, m$;

- each job $J_{i}$ is composed of $n_{j}$ operations: $O_{j}=\left\{o_{j k}\right\}$, $k=1, \ldots, n_{j}$;

- each operation can be processed on (more) different sets of resources $S_{j k l} \in R$; $l$ determines the number of different sets;

- the processing time of each operation $o_{j k l}$, using resource set $S_{j k l}$, is defined as $T_{j k l}$;

- precedence constraints are used to define that one job has to be performed before another.

Using this definition, the following assumptions have to be considered.

- Resources are always available and never break down.

- Each resource can process a limited number of operations. This limitation is defined by the capacity of resources.

- Operations are non pre-emptive.

- When an operation is performed, it is desirable to free the resources so that they can become available as soon as possible. Intermediate buffers between processes are common solutions.

- Processing times are deterministic and known in advance.

- Work orders define the quantity of desired products and the starting times. Orders that are synchronized in time are considered jointly.

- In the case where a job requires sub-products, for each of the additions, sub-jobs are to be defined.

\subsection{The modelling of production activities}

First, a method of describing the production-system activities with timed Petri nets using the holding-duration representation of time is presented. The places represent resources and jobs/operations, and the transitions represent decisions or rules for resource assignment/release and for starting/ending jobs.

To make a product, a set of operations has to be performed. We can think of an operation as a set of events and activities. Using a timed $\mathrm{PN}$, events are represented by transitions and activity is associated with the presence of a token in a place.

An elementary operation can be described by one place and two transitions (see figure 1). When all the input conditions are met (raw material and resources are available) the event that starts the operation occurs, $t_{1}$. This transition also determines the processing time of an operation. During that time the created token is unavailable in place $p_{2}$ and the operation is being executed. After that time the condition for ending the operation is being satisfied and $t_{2}$ can be fired. Place $p_{1}$ is not a part of the operation, it determines the input condition.

When parallel activities need to be described the Petri-net structure presented in figure 2 is used. The time delays of the transitions $t_{11 \text { in }}$ and $t_{12 \text { in }}$ define the duration of each operation. An available token in place $p_{11 \text { out }}\left(p_{12 \text { out }}\right)$ indicates that the operation is finished. Transition $t_{1}$ is used to synchronize both operations.

An operation might need resources, usually with a limited capacity, to be executed; this is illustrated in figure 3. Place $p_{R I}$ is used to model a resource. Its capacity is defined by the initial marking of that place. The resource is available to process the operation if there are enough available tokens in it. When the resource is used at the start of the operation the unavailable token appears in place $p_{1 \text { op. }}$. After the time defined by transition $t_{1 \text { in }}$ the token becomes available, $t_{1 \text { out }}$ is fired, and the resource becomes free to operate on the next job. For this reason, zero time

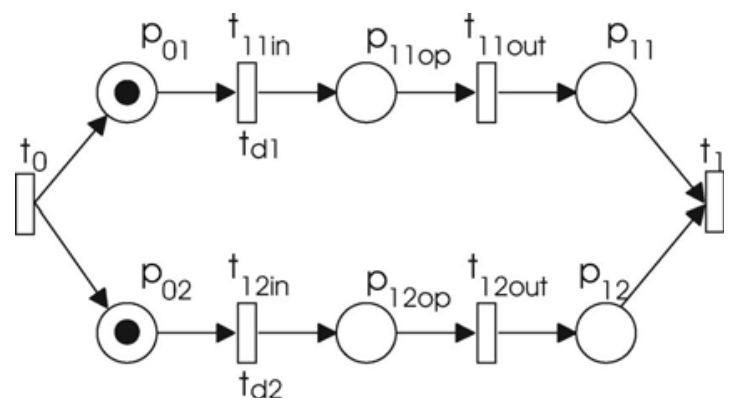

Figure 2. Two parallel operations.

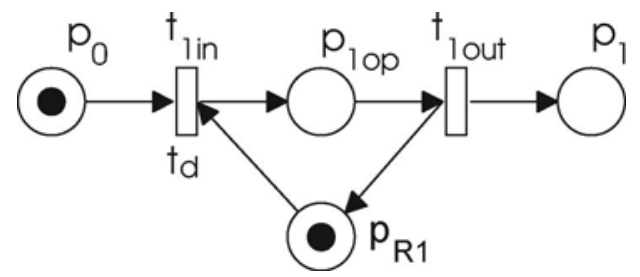

Figure 3. Operation that uses a resource with finite capacity. 
needs to be assigned to the transition $t_{\text {1out }}$. An additional place $p_{1}$ models the control flow. When the token is present in this place, the next operation can begin.

A particular operation can often be performed on different (sets of) resources with different availability, and the time duration can be different on each set of resources. An example where an operation can be executed on two different sets of resources is shown in figure 4 . If the operation chooses resource $R_{3}$, its time duration is determined by the transition $t_{2 \mathrm{in}}=t_{\mathrm{d} 2}$. Otherwise, the set of resources, composed of $R_{1}$ and $R_{2}$, is being selected and its operation time is defined by $t_{1 \text { in }}=t_{\mathrm{d} 1}$.

There are common situations where more operations use the same resource, e.g. an automated guided vehicle (AGV) in a manufacturing system or a mixing reactor in a batch system. This can be modelled as shown in figure 5 .

Precedence constraints are used to define technological limitations and the sequence of operations. An example of two successive operations is shown in figure 6 , depicted as $O p 1$ and $O p 2$. In this figure an example of technological limitations is also shown. Here, the situation where

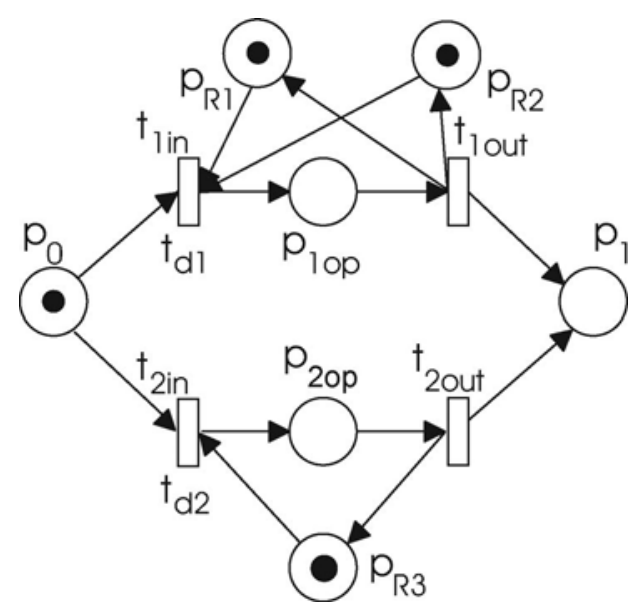

Figure 4. Operation that can be performed on two different sets of resources.

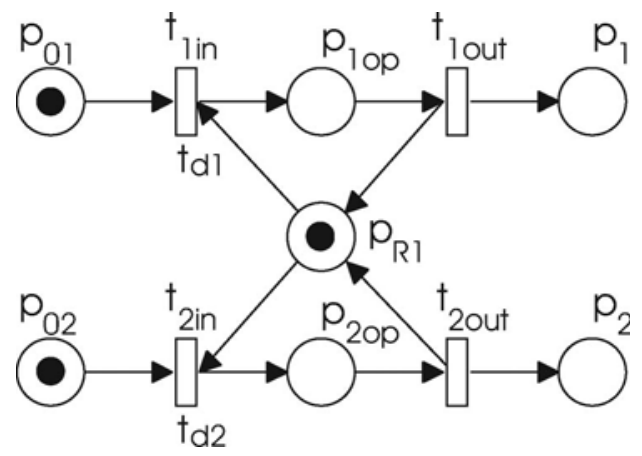

Figure 5. Shared resource. operation $O p 1$ precedes operation $O p 3$ is considered. For this purpose an additional place $p_{\mathrm{pr} 1}$ is inserted between the transition $t_{1 \text { out }}$ (ending $O p 1$ ) and the transition $t_{3 \text { in }}$ (starting $O p 3$ ). The weight $n$ of the arc that connects $p_{\mathrm{pr} 1}$ to $t_{2 \text { in }}$ prescribes how many items need to be produced by the first operation before the second operation can begin.

\subsection{Modelling using the data from production-management systems}

The most widely used production-management information system in practice is MRP II. Data stored in these systems can be used to build up a detailed model of the production system with Petri nets.

3.3.1. BOM. The BOM is a listing or description of the raw materials and items that make up a product, along with the required quantity of each. The BOM used in this work is defined as

$$
B O M=(R, E, q, \text { pre })
$$

where $R=r_{1}$ is a root item; $E=\left\{e_{1}, \ldots, e_{i}\right\}$ is a finite set of sub-items; $q: E \rightarrow \mathbb{N}$ is the function that defines the quantities for each sub-item $e_{i}$. q represents an $i \times 1$ column vector whose $i$ th entry is $q\left(e_{i}\right)$; and pre $:(E \times E) \rightarrow\{0,1\}$ is a precedence-constraints function. It defines the precedenceconstraints matrix pre, where pre $(i, j)=1$ indicates that the $i$ th item precedes the $j$ th item. It can also be interpreted as a directed graph.

$R$ is a root item and represents the product that is composed of sub-items described by $e_{i} \in E$. The number of required sub-items is determined by the vector $\mathbf{q}$. When any sub-item has to be produced before another, the precedence graph pre is used to define it. All the sub-items have to be finished before the operation for the subsequent sub-items can begin. A required property of pre is that only zero values can be on its diagonal, i.e. a sub-item cannot precede itself. An item is never allowed to become (indirectly) a component of itself. In other words, if the BOM structure is seen as a directed graph, this graph should be cycle-free (Wortmann 1995).

If any of the sub-items $e_{i}$ are composed of any other subitems, the same BOM definition is used to describe its dependencies. The items at the highest level of this structure represent a finished product, and those at the lower level represent raw materials. The items that represent raw materials do not have a BOM.

Table 1 shows an example of a BOM describing the production of product $I$, which is composed of two components, i.e. three items of $J$ and and two items of $K$. From the precedence-constraint matrix it is clear that all of the items $J$ have to be completed before the production of item $K$ can begin. 


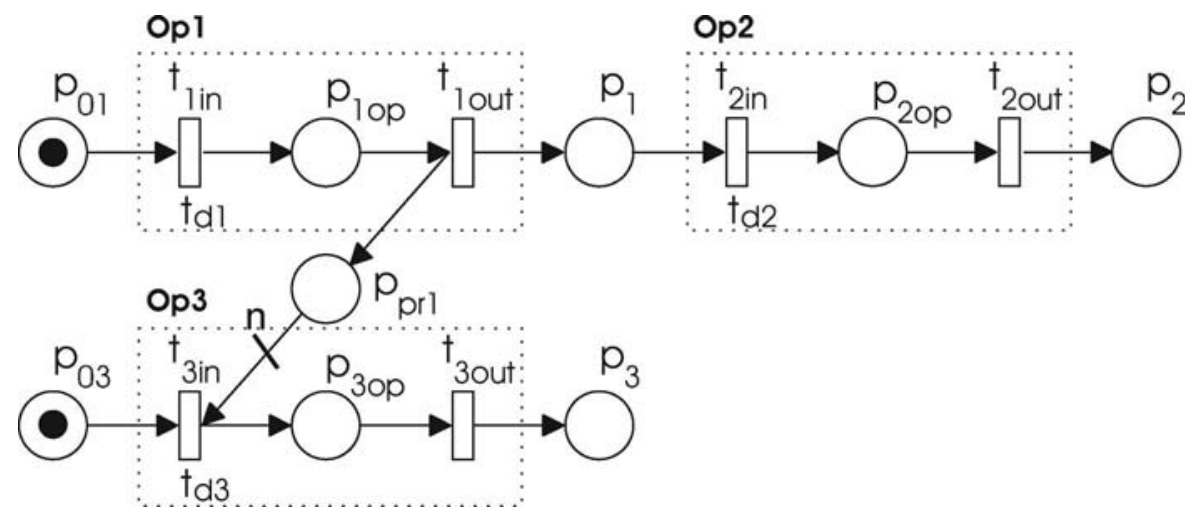

Figure 6. Precedence constraint.

Table 1. Example of the BOM structure.

\begin{tabular}{llclr}
\hline Item & Sub-item & Quantity & \multicolumn{2}{c}{$\begin{array}{c}\text { Precedence } \\
\text { constraint }\end{array}$} \\
\hline$I$ & $J$ & 3 & 0 & 1 \\
& $K$ & 2 & 0 & 0 \\
\hline
\end{tabular}

The mathematical representation of the BOM of item $I$ would be

$$
\begin{aligned}
& B O M=(R, E, \mathbf{q}, \text { pre }), \quad \text { where } R=\{I\}, \\
& E=\{J \quad K\}, \mathbf{q}=\left[\begin{array}{ll}
3 & 2
\end{array}\right] \quad \text { and pre }=\left[\begin{array}{ll}
0 & 1 \\
0 & 0
\end{array}\right] .
\end{aligned}
$$

To start, let us assume that, for each item from the BOM, only one operation is needed. As stated before, each operation can be represented with one place and two transitions (figure 1). To be able to prescribe how many of each item is required, the transition $t_{\text {Rin }}$ and the place $p_{\text {Rin }}$ are added in front, and $p_{\text {Rout }}$ and $t_{\text {Rout }}$ are added behind this operation. The weight of the arcs that connect $t_{\mathrm{Rin}}$ with $p_{\text {Rin }}$ and $p_{\text {Rout }}$ with $t_{\text {Rout }}$ are determined by the quantity $q_{0}$ of the required items. In this way an item $I$ is represented by a Petri net as defined in figure 7 .

The finished product is defined by a structure of BOMs. In this way the construction of the overall Petri net is an iterative procedure that starts with the root of the BOM and continues until all the items have been considered. If the item requires any more sub-assemblies (i.e. items from a lower level) the operation, the framed area of the PN structure presented in figure 7 , is substituted by lower-level items. If there are more than one sub-items, they are given as parallel activities.

The substitution of an item with sub-items is defined as follows.

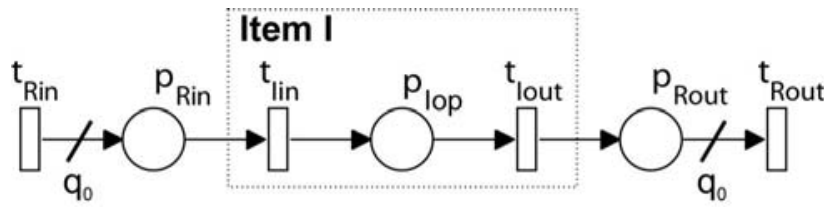

Figure 7. PN structure representing one item in the BOM.

- Remove the place $p_{X o p}$ and its input/output arcs.

- Define the PN structure for sub-components, as it is defined with a BOM: function $P N=\operatorname{place} P N(R, E$, $q$, pre). Consider the precedence constraints.

- Replace the removed place $p_{X o p}$ by the sub-net defined in the previous step. The input and output transitions are merged with the existing ones: $P N=\operatorname{insert} P N(P N, P N 1)$. Structure PN1 is inserted in the main structure PN.

The result of building the PN model of this example (table 1 ) is given in figure 8 .

3.3.2. Routings. For each item that can appear in the production process, and does not represent a raw material, a routing is defined. It defines a sequence of operations, each requiring processing by a particular machine for a certain processing time. The routing information is usually defined by a routing table. The table contains a header, where the item that is being composed is defined, and the lines, where all the required operations are described. For each operation, one line is used.

As an example, the routing table for item $K$ is presented in table 2. Two operations are needed to produce this item. The first can be performed on two different resources, where the processing of each demands a different processing time. This is followed by the next operation, which needs two sets of resources: one resource of $R 1$ and three of 


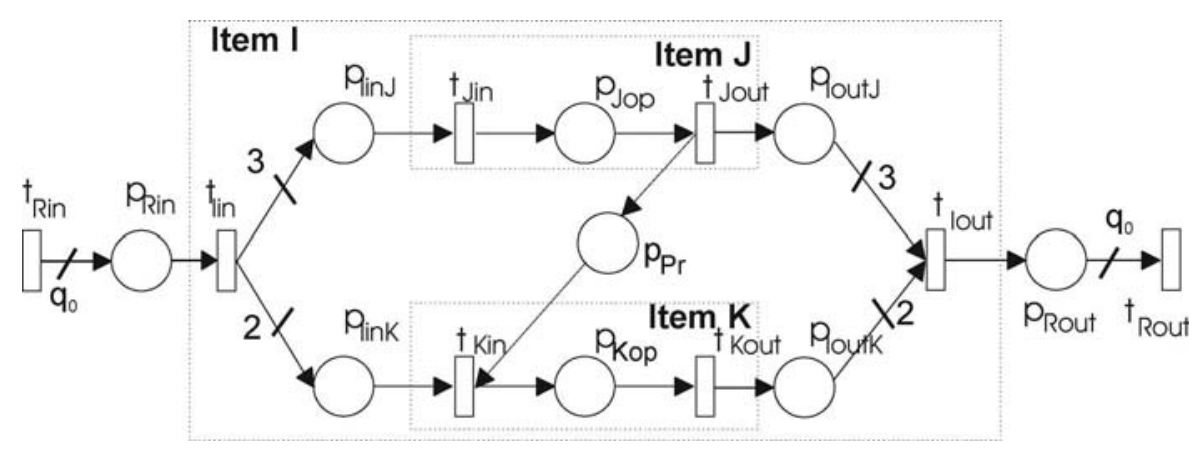

Figure 8. BOM structure defined by PN.

Table 2. Routing of product $K$.

\begin{tabular}{ccc}
\hline$J$ & Duration & Resources \\
\hline$O p 10$ & $10 \mathrm{~s} / 9 \mathrm{~s}$ & $R 1 / R 2$ \\
$O p 20$ & $20 \mathrm{~s}$ & $R 1,3 R 3$ \\
\hline
\end{tabular}

R3. A similar notation can be used for the other possible cases. Within the routing table, concurrent operations are considered as a single operation with a parallel internal structure, as shown in figure 2.

The implementation of the routing data in one component of a BOM is defined as follows.

- Remove the place $p_{X o p}$ and its input/output arcs.

- Define a PN structure for the sub-components, as it is defined with routing data: function $P N 1=$ construct $P N(P N$, datRoute).

- Replace the removed place $p_{X o p}$ by the sub-net defined in the previous step. The input and output transitions are merged with the existing ones: $P N=\operatorname{insert} P N(P N, P N 1)$. Structure PN1 is inserted in the main structure PN.

Each operation that appears in the routing is placed in the model using the function construct $P N()$. From the routing table the function yields the corresponding sequence of production operations and for each operation build a timed Petri net as defined in section 3.2. All the placed operations are connected as prescribed by the required technological sequence, and each operation is assigned to the required places representing appropriate resources.

The PN structure in figure 9 is achieved if the sequence of operations described by the routing table (table 2) is modelled. The resulting PN structure is inserted into the main PN model using the function insert $P N()$.

The routings are sub-models that are inserted (by substitution, as defined previously) into the main model defined by the BOM structure. However, some activities of any sub-item may also be described with a BOM, i.e. in the case they are composed of semi-products. The construction of the overall Petri-net model can be achieved by combining all of the intermediate steps.

3.3.3. Procedure for building the PN model. The work order (WO) determines which and how many of the finished products have to be produced. Each product can be represented by a Petri-net model, shown in figure 7, where one place is added in front and one at the end of the structure to determine the start and end of the work. The weight of the arc that connects $t_{\text {Rin }}$ and $p_{\text {Rin }}$ determines the number of required products. To be able to consider different starting times for different quantities of one product the general structure shown in figure 10 is used. The clocks, which are assigned to the tokens that are in starting places, determine the starting time of every batch of products.

The modelling procedure can be summarized by the following algorithm:

Algorithm 1

$$
\begin{aligned}
& {[\mathrm{R}, \mathrm{q}, \mathrm{st}]=\operatorname{read} W O()} \\
& \text { For } \mathrm{i}=1 \text { to } \operatorname{length}(\mathrm{R}) \\
& \mathrm{E}=\operatorname{readBOM}(\mathrm{R}(\mathrm{i})) \\
& \mathrm{PN}=\operatorname{place} P N(\mathrm{R}(\mathrm{i}), \mathrm{E}, \mathrm{q}(\mathrm{i}),[], \mathrm{st}(\mathrm{i}), \mathrm{x} 0, \mathrm{y} 0) \\
& \mathrm{PN}=\operatorname{routing}(\mathrm{PN}, \mathrm{R}(\mathrm{i})) \\
& \text { end }
\end{aligned}
$$

First, the data concerning the WO are read. The products that are needed to be produced are given in $R$; in vector $\mathbf{q}$ the quantities of the desired products are passed; and vector st is used to determine the starting time of each product. For each product the Petri-net structure, shown in figure 10 , is determined and placed in the model. The step when the routing () is called is described in more detail by algorithm 2:

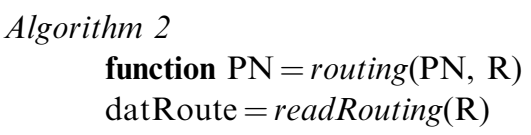




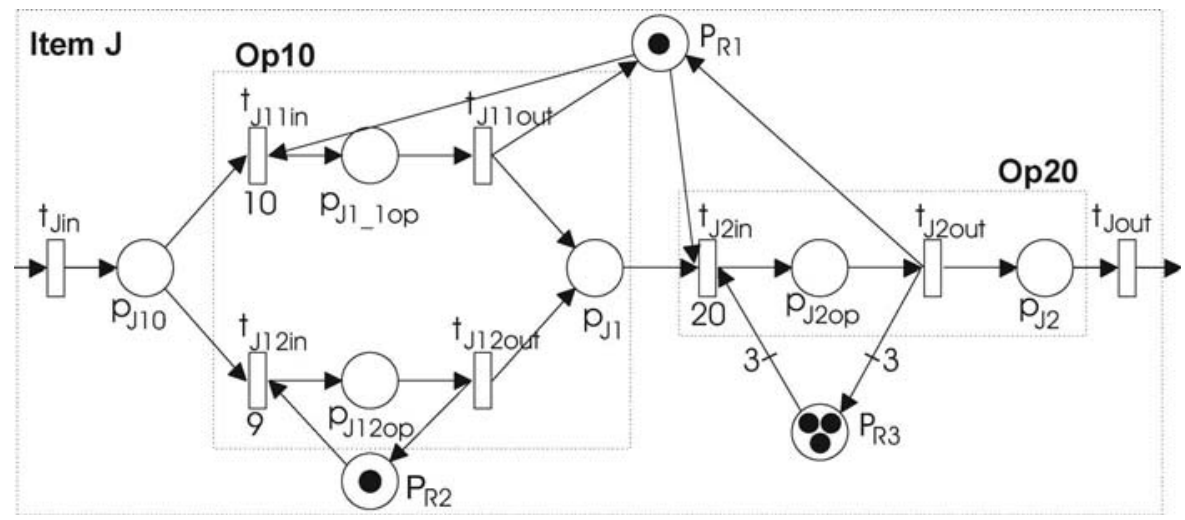

Figure 9. Routing of product $K$ modelled with a timed Petri net.

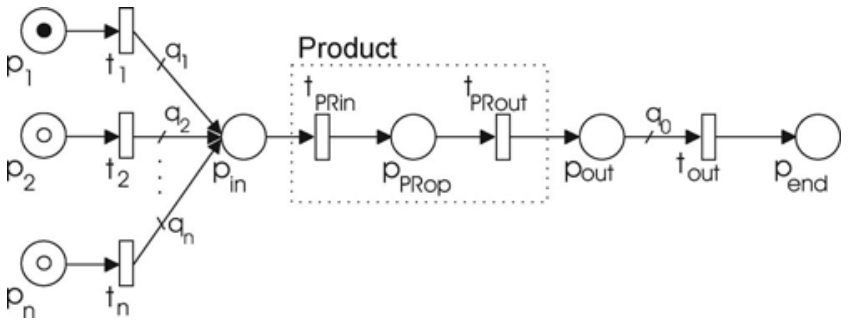

Figure 10. Petri-net structure of a work order.

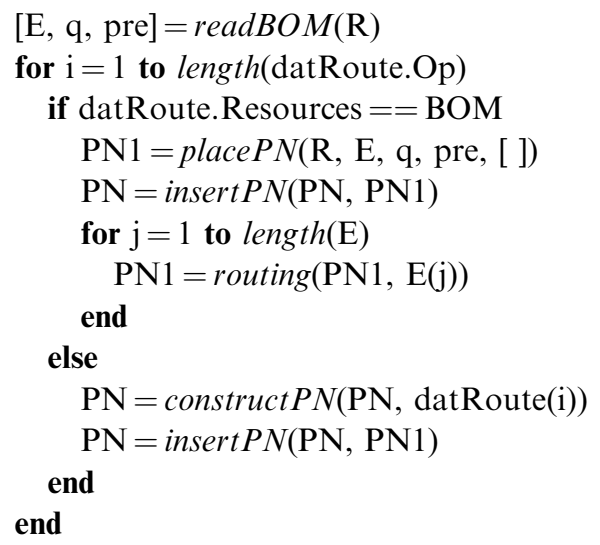

First, the routing and the BOM data are read from the database. For each operation that comprises the routing, the algorithm checks whether it is made up of sub-item(s) or it is an operation. In the first case, the function place $P N()$ is used to determine the PN structure of the given structure BOM. Precedence constraints are added if they exist. With the function insert $P N()$ the resulting subnet is inserted into the main PN structure. If the operation represents the production operation, the function constructPN() is called. With it, basic elements (figures 1-6) are recognized, joined together and placed in the model, again using the function insertPN(). All data concerning resources and time durations are acquired from the routing table. The described algorithm has been implemented in Matlab.

3.3.4. Verification. When the model is built up, it should be verified to see whether it reflects the system operation as defined in the MRP II system. Some interesting properties of the model can be checked with the P-invariant analysis. Several P-invariants can be identified in the model. For example, every distinguishable product route results in a P-invariant. Another P-invariant appears as a result of a precedence constraint. Every shared resource also adds a P-invariant. So the number of invariants is defined by the sum of resources, the number of product routes and the number of precedences that are present in the model. It can be stated that the weighted sum of tokens that belongs to every P-invariant, which is a consequence of a resource, is equal to the capacity of that resource. The weighted sum of all other invariants is defined by the common number of batches of demanded product.

\section{Simulator-scheduler}

A Petri-net model can be used to verify and validate the logical properties of the behaviour of modelled processes. The analysis can be based either on the reachability (coverability) tree or calculation of the invariants. For complex models, such an analysis is computationally expensive. Instead, certain properties can be estimated by a simulation. Using a simulation the evolution of the marking through time can be observed. Different heuristic rules can then be introduced when solving situations in which conflicts occur. In this way, different evolutions of the Petri net are usually possible. When the marking of the places that represent resources is being considered, the schedule of process operations can be observed, i.e. when, 
and using which resource, a job has to be processed. Usually, different rules are needed to improve different predefined production objectives (makespan, throughput, production rates, and other temporal quantities).

To demonstrate the practical applicability of the proposed modelling method for the purposes of scheduling we built a simulator in Matlab that takes a Petri-net model generated by the above algorithm. The simulator allows different priority dispatching rules to be implemented. With the simulation a marking trace of a timed Petri net can be achieved. The marking trace of places that represent resources is characterized as a schedule. The simulator is able to deal with situations in which a conflict occurs, the conflict being solved by a decision maker. By introducing different heuristic dispatching rules (priority rules) decisions can be made easily. In this way, only one path from the reachability graph is calculated, which means that the algorithm does not require a lot of computational effort. Depending on the given scheduling problem a convenient rule should be chosen.

The procedure of each simulation step computes a new state $s_{k+1}=\left(\mathbf{m}_{k+1}, \mathbf{n}_{k+1}, r_{k+1}\right)$ of a timed Petri net in the next calculation interval.

(i) Obtain the marking of the classical (untimed) Petri net, i.e. only available tokens are considered. Calculate the corresponding firing vector $\mathbf{u}_{\mathbf{k}}$ of the untimed Petri net. At this point a conflict resolution is also applied.

- $\mathbf{m}_{k} \rightarrow \mathbf{u}_{\mathbf{k}}$.

(ii) If no transition is being fired $\left(\mathbf{u}_{\mathbf{k}}=0\right)$, the time passes on - the values of the local clocks are decreased by the value of the smallest local clock.

- $\mathbf{M}_{k}=\mathbf{m}_{k}+\mathbf{n}_{k}$,

- $T_{s}=\min p_{i} \in P\left(\min _{l}\left(\mathbf{r}_{k}\left(p_{i}\right)[l]\right)\right), \quad \mathbf{r}\left(p_{i}\right)$ is not empty,

- $\quad \mathbf{r}_{k+1}\left(p_{i}\right)=\mathbf{r}_{k}\left(p_{i}\right)-T_{s}, \forall r\left(p_{i}\right)[l], \forall p_{i} \in P$ and $\mathbf{r}\left(p_{i}\right)$ is not empty - remove clocks for tokens that became zero,

- $\quad n_{k+1}\left(p_{i}\right)=\operatorname{dim}\left(\mathbf{r}_{k+1}\left(p_{i}\right)\right), \forall p_{i} \in P$ and $\mathbf{r}\left(p_{i}\right)$ is not empty,

- $\mathbf{m}_{k+1}=\mathbf{M}_{k}-\mathbf{n}_{k+1}$.

(iii) In other cases $\left(\mathbf{u}_{\boldsymbol{k}} \neq 0\right)$, the time remains the same. The firing vector $\mathbf{u}_{\boldsymbol{k}}$ defines the new state of the timed Petri net, i.e. the distribution of tokens over the places and the values of local clocks corresponding to newly created tokens.

- $\mathbf{M}_{k}=\mathbf{m}_{k}+\mathbf{n}_{k}$,

- $\mathbf{M}_{k+1}=\mathbf{M}_{k}+(\mathbf{O}-\mathbf{I}) \cdot \mathbf{u}_{\boldsymbol{k}}$,

- $n_{k+1}\left(p_{i}\right)=n_{k}\left(p_{i}\right)+O\left(p_{\mathrm{i}}, t_{j}\right) \cdot u_{k j}, \forall p_{i} \in P$ and $\forall t_{j} \in$ $\{t \in T: f(t)>0\}$,

- $\quad \mathbf{r}_{k+1}\left(p_{i}\right)=\mathbf{r}_{k}\left(p_{i}\right) ; \mathbf{r}_{k+1}\left(p_{i}\right)[h]=f\left(t_{j}\right)$, for $h=n_{k}\left(p_{i}\right)+$ $1, \ldots, n_{k+1}\left(p_{i}\right)$,

- $\mathbf{m}_{k+1}=\mathbf{M}_{k+1}-\mathbf{n}_{k+1}$.
In step (i) the firing vector $\mathbf{u}_{\boldsymbol{k}}$ is determined. In the case of conflict it has to determine which transition to fire from among all those that are enabled. Different heuristics can be applied at this point. In step (ii), when no transitions are enabled, first the sum of the available and unavailable tokens is defined $\left(\mathbf{M}_{\mathbf{k}}\right)$. Next the value of the smallest local clock has to be recognized $\left(T_{s}\right)$. The values of every clock are then decreased for $T_{s}$. If the value of any clock becomes zero, this clock has to be destroyed. As the number of unavailable tokens has changed, vector $\mathbf{n}$ has to be redefined in the next stage. Vector $\mathbf{M}_{\mathbf{k}}$ can then be used to determine $\mathbf{m}_{k+1}$, as the number of tokens in a place does not change. Step (iii) deals with the situation when firing has to be performed as determined by firing vector $\mathbf{u}_{\mathbf{k}}$. Again, first the sum of the available and unavailable tokens is defined $\left(\mathbf{M}_{\mathbf{k}}\right)$. Firing vector $\mathbf{u}_{\mathbf{k}}$ determines the new amount of all tokens $\mathbf{M}_{\mathbf{k}+\mathbf{1}}$. The new number of unavailable tokens can be calculated separately. In the next stage the clocks for each token have to be determined $\mathbf{r}_{k+1}\left(p_{i}\right)$. While previous local clocks remain the same, new local clocks have to be defined for every newly created token. The initial state of the clock is determined by the delay of the transition that created that token. The vector of available tokens can now easily be determined from $\mathbf{M}_{k+1}$ and $\mathbf{n}_{k+1}$.

The simulation is finished when there are no unavailable tokens in the model or the global clock reaches the predefined stop time.

In this algorithm the firing of the transitions is instantaneous. This is solved by the fact that time is not passed on when any transition is enabled. It is assumed that the situations where the enabling (firing) of a transition at one time instant is cycling are prohibited. This kind of situation would only occur in the case of incorrect modelling.

After the simulation is finished the marking over time can be observed. A Gantt chart can be produced if evolution of the marking in the places that represent the resources is observed. From the chart the schedule of the modelled process can be obtained.

\section{Case study: the production of furniture fittings}

The applicability of our approach will be demonstrated on a model of a production system where furniture fittings are produced. The production system is divided into a number of departments. The existing information-technology systems include a management system, which is used to plan the production, and a supervisory system, which is used to supervise the production process. To implement a detailed schedule, how the work should be done, an additional scheduling system should be implemented. The scheduling can be performed using timed Petri nets. The data needed to produce a Petri-net model can be retrieved from the 
existing information systems. In the presented model, only a small part of the production process will be considered.

The process under consideration is an assembly process where different finished products are assembled from a number of sub-items. During the production process, different production facilities are used to produce sub-items and finished products. The production facility considered in this example is shown in figure 11 . The process route of each finished product starts with a punching machine. The process continues through different resources, such as assembly lines, a paint chamber, and galvanization lines. At the final stage there is another assembly line where the finished products are assembled and packed.

In the considered problem, four different types of products are produced: 'Corner clamp L', 'Corner clamp R', 'Clamp holder' and 'Angle-bar'. The request for what and how many products to produce is given by work orders. Each work order also has its starting time. Table 3 lists the work orders considered in our case.

Each product is composed of one or more sub-assembly items. The structure of all products is described by the bill of materials (table 4). When there are no precedence constraints between sub-items, all the elements of the precedence-constraints matrix are 0 , and the matrix is simply represented by [0].

To build a particular item from the BOM list, some process steps are needed. These steps are described by routing data and are given in tables 5-7. A description of the data presented in the table will be given later during the modelling procedure.

The final product of one production stage represents the input material for the next stage. In this way the scheduling procedure should ensure that work orders are timely adjusted and thus ensure that, at each stage, sufficient semi-products are produced. This kind of schedule is feasible. Using different heuristic rules it is possible to obtain a schedule that satisfies different objectives.

\subsection{Modelling}

Data from the BOM and the routings were used to build a Petri-net model. The development of the model will be presented for the part of the production where the left corner clamp is produced. As we can see from table 3 , there are two work orders: the first has to start immediately with its production, while the starting time of the second is 80 time units later. When we apply the first step of our algorithm, the PN structure, shown in figure 12, is obtained. The production of different amounts of products starts at different times. Starting times are defined by the tokens in the starting places $p_{\mathrm{WOCL} 1}$ and $p_{\mathrm{WOCL} 2}$, and the quantity of each is defined by the weights of the corresponding arcs.

From table 5 the routing data concerning the product 'Corner Clamp L' $(C L)$ are read. The first operation in this table $(O p 1)$ shows that the sub-items should be produced first as prescribed by 'BOM_CCL'. This BOM is defined in table 4 , and, as we can see, four different sub-items are needed ('Angle-bar with holder L', 'Clamp', 'Nut' and 'Screw'). It is clear from the table that, for each item, a designation character is given with its item name. These characters are used in the Petri-net model to indicate the item. When these subitems are produced, the production proceeds with an assembly - Op2. This situation is demonstrated by the Petri-net structure shown in figure 13 .

In the following, the algorithm recognizes the routing data for each of these sub-items. The routing data needed to build the sub-items of a 'Corner Clamp L' are given in table 5 . There is one operation needed to produce the subitem 'Screw', two operations to produce 'Nut' and 'Clamp', and three operations to produce the 'Angle-bar with holder L'. Using these data the structure as defined in figure 14 is achieved. Some denotations of the transitions and places are omitted to achieve a clearer representation of the model. Also, the nodes that designate the start and end of production are not shown.

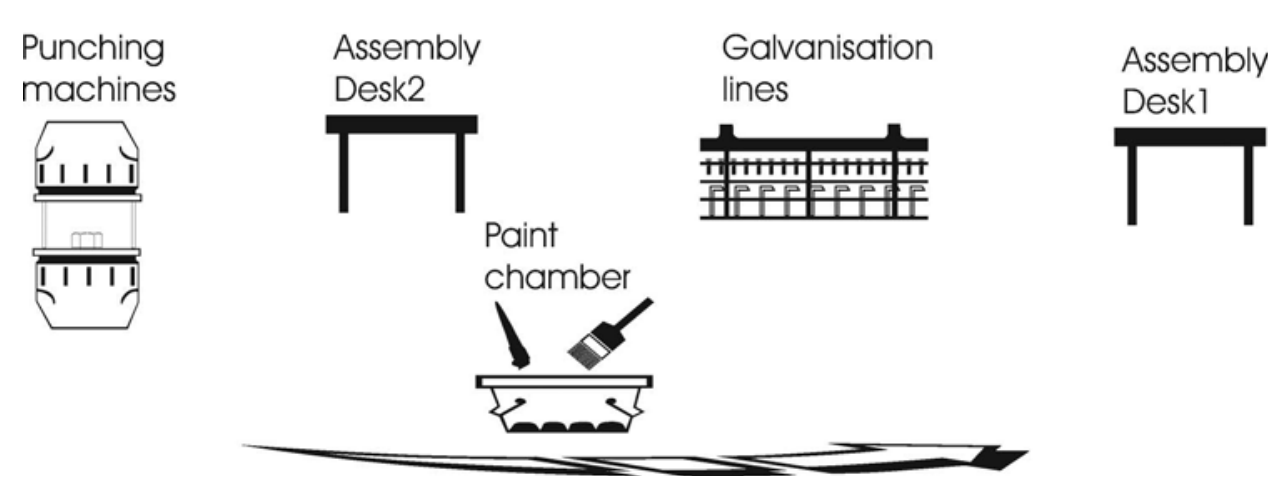

Figure 11. Production plant. 
Table 3. Work orders for required products.

\begin{tabular}{lccc}
\hline Product & Code & Quantity & Start time \\
\hline Corner clamp L & CL & 2 & 0 \\
Corner clamp L & CL & 2 & 80 \\
Corner clamp R & CR & 2 & 0 \\
Clamp holder & CH & 2 & 0 \\
Angle-bar & AB & 2 & 0 \\
\hline
\end{tabular}

Table 4. Bill of materials.

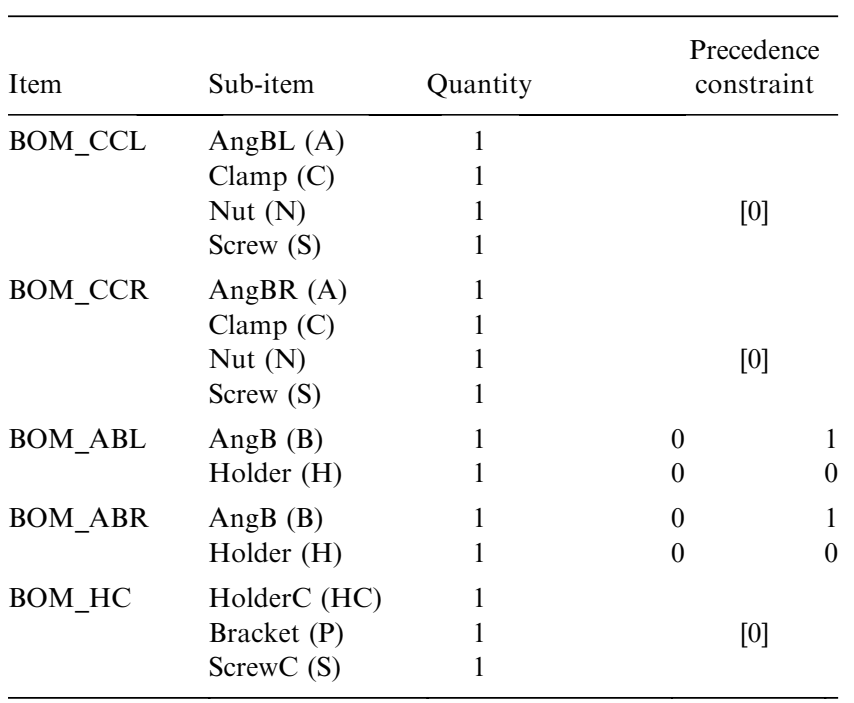

Table 5. Routings of each component from product 'Corner clamp L'.

\begin{tabular}{lccl}
\hline & Operation & Duration & Resources \\
\hline CL & $O p 1$ & & BOM_CCL \\
& $O p 2$ & 10 & Desk1 \\
AngBL & $O p 11$ & & BOM_ABL \\
& $O p 12$ & 10 & Desk2 \\
& $O p 13$ & 20 & Galvanisation1 \\
Clamp & $O p 21$ & 3 & V1 \\
& $O p 22$ & 20 & Galvanisation2 \\
Nut & $O p 31$ & 2 & V1 \\
\multirow{2}{*}{ Screw } & $O p 32$ & 20 & Galvanisation2 \\
AngB & $O p 41$ & 20 & Galvanisation2 \\
Holder & $O p 111$ & 8 & V1 \\
& $O p 221$ & 8 & V2 \\
\hline
\end{tabular}

As can be seen the production of angle-bar with holder ('AngBL') requires another two sub-items ('AngB' and 'Holder'). The precedence-constraint matrix defines that item 'AngB' should be produced before 'Holder'. When all these details are included in the model we obtain the Petrinet structure presented in figure 15 .

The same procedure was performed to model the production of all the other products, and the Petri-net
Table 6. Routings of each component from product 'Clamp holder'.

\begin{tabular}{lccl}
\hline & Operation & Duration & Resources \\
\hline $\mathrm{CH}$ & $O p 1$ & & BOM_HC \\
HolderC & $O p 2$ & 5 & Desk1 \\
& $O p 11$ & 15 & Galvanisation2 \\
Bracket & $O p 12$ & 10 & Paint Chamber \\
ScrewC & $O p 21$ & 10 & Paint Chamber \\
\hline
\end{tabular}

Table 7. Routings of each component from product 'Anglebar'.

\begin{tabular}{cccl}
\hline & Operation & Duration & Resources \\
\hline $\mathrm{AB}$ & $O p 1$ & 8 & V2 \\
& $O p 2$ & 15 & Galvanisation1 \\
& $O p 3$ & 5 & Desk1 \\
\hline
\end{tabular}

model given in figure 16 represents the given scheduling problem.

Finally, the resulting model is verified using P-invariant analysis. With this analysis we can determine 23 P-invariants. Seven of them are related to resources, two invariants refer to the precedence constraints and there are 14 invariants that result from every distinguishable product route. From this we can conclude that the resulting model reflects the system operation as defined by the MRP II system.

\subsection{Results}

The scheduling problem was mapped onto timed Petri nets, and the assembly process was modelled with timed Petri nets. The simulator/scheduler was used to evaluate the different schedules of the tasks that are needed to produce the desired number of finished products. We tested different priority rules (SPT, LPT, etc.) and different schedules were achieved. The schedule allows an easy visualization of the process and ensures that sufficient raw materials are available at the right time. It respects all the production constraints and the duration of the whole process can be identified. The shortest duration, i.e. 220 time units, would be achieved if the schedule was determined with the SPT priority rule (see figure 17).

The generated model can also be used in conjunction with other PN-based scheduling algorithms. As an example, a comparison with a more advanced PN scheduling method was made using the same timed PN model of the assembly process. The PN-based heuristic search method of Lee and DiCesare (1994) was programmed in Matlab and used with a slightly modified heuristic function, as proposed by Yu et al. (2003b): $h(m)=-w^{\prime} \cdot \bar{O}_{p} \cdot \operatorname{depth}(m)$. A search 


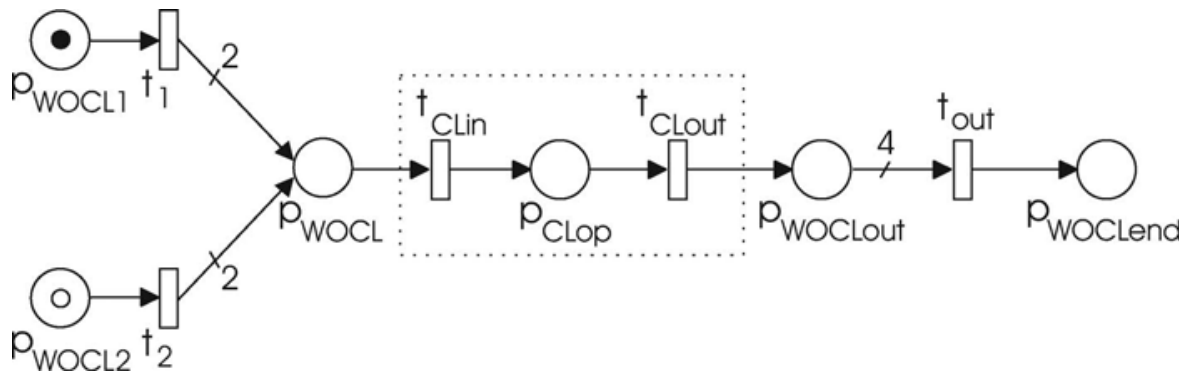

Figure 12. Initial PN model of product 'Corner clamp L'.

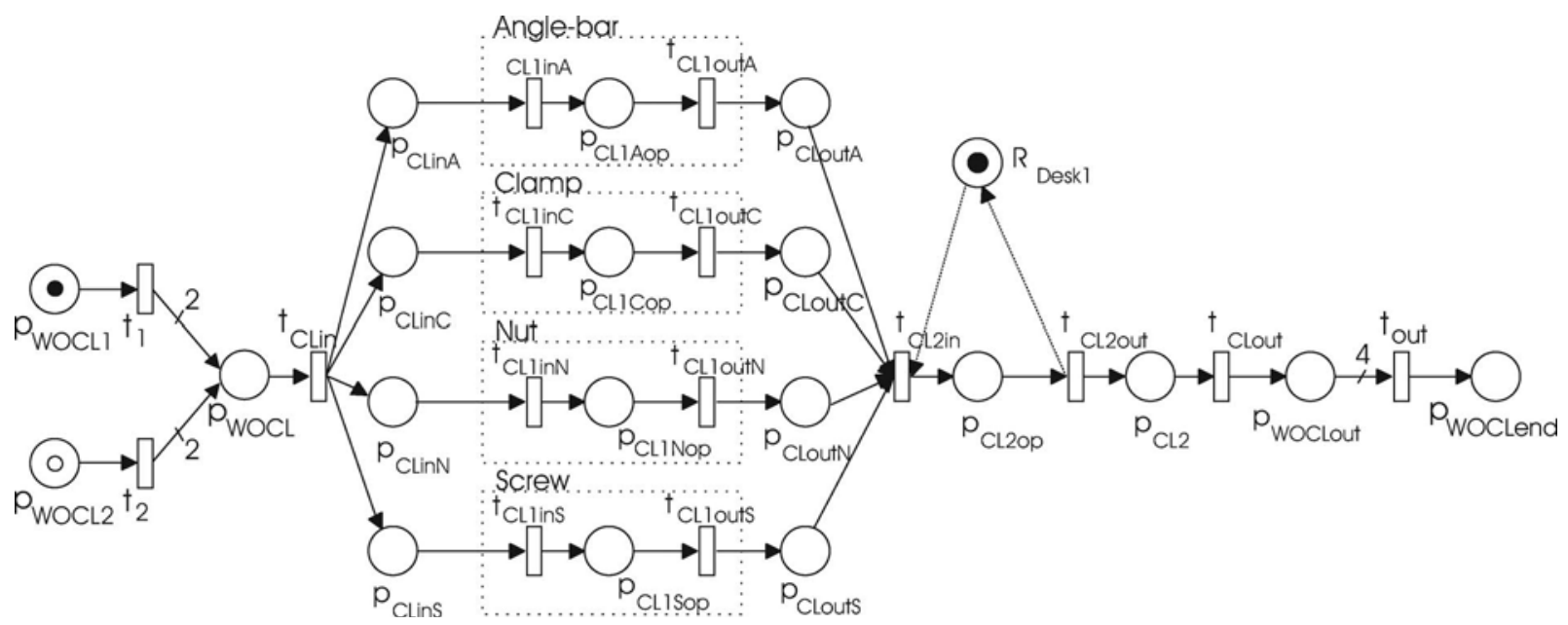

Figure 13. 'Corner clamp L' is composed of four sub-items.

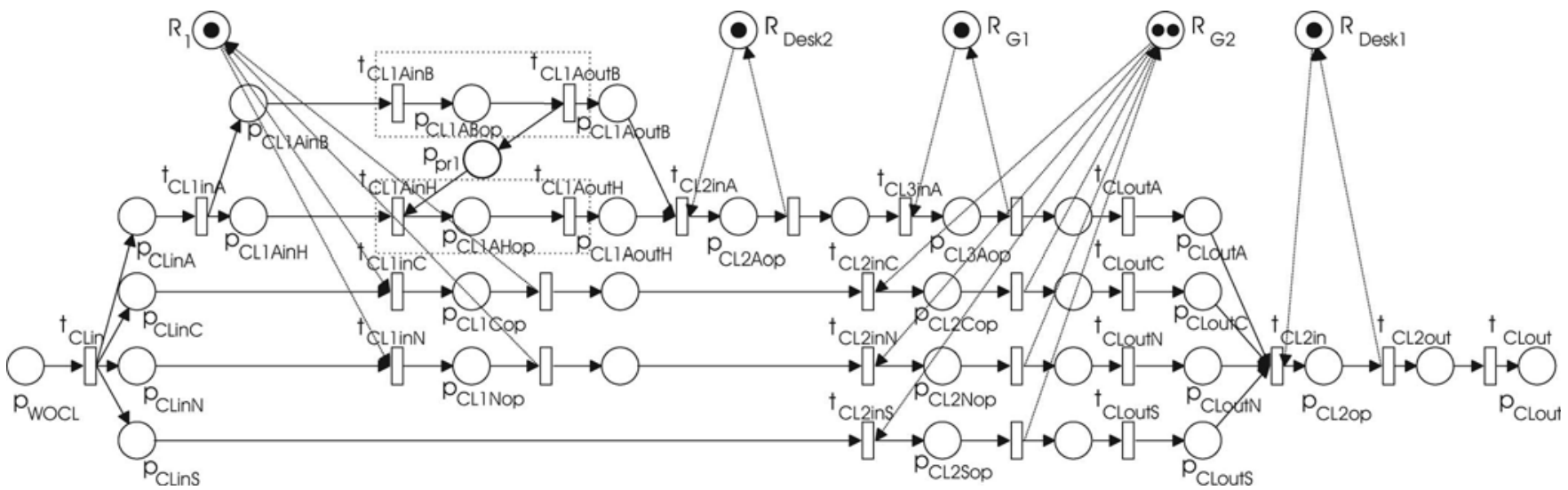

Figure 14. Angle-bar with holder requires another two sub-items.

with parameter $w^{\prime}$ set to 0.5 resulted in the same makespan as the SPT rule.

The same problem was solved using the commercial scheduling tool Preactor. Also, in this way, the model of a production system was achieved using the data from the database of a management information system. As the precedence constraints, e.g. item 'AngB' precedes 'Holder', in this configuration of the tool could not be defined, this has to be done manually on the planning desk. Here, the blocked-time approach is used (Enns 


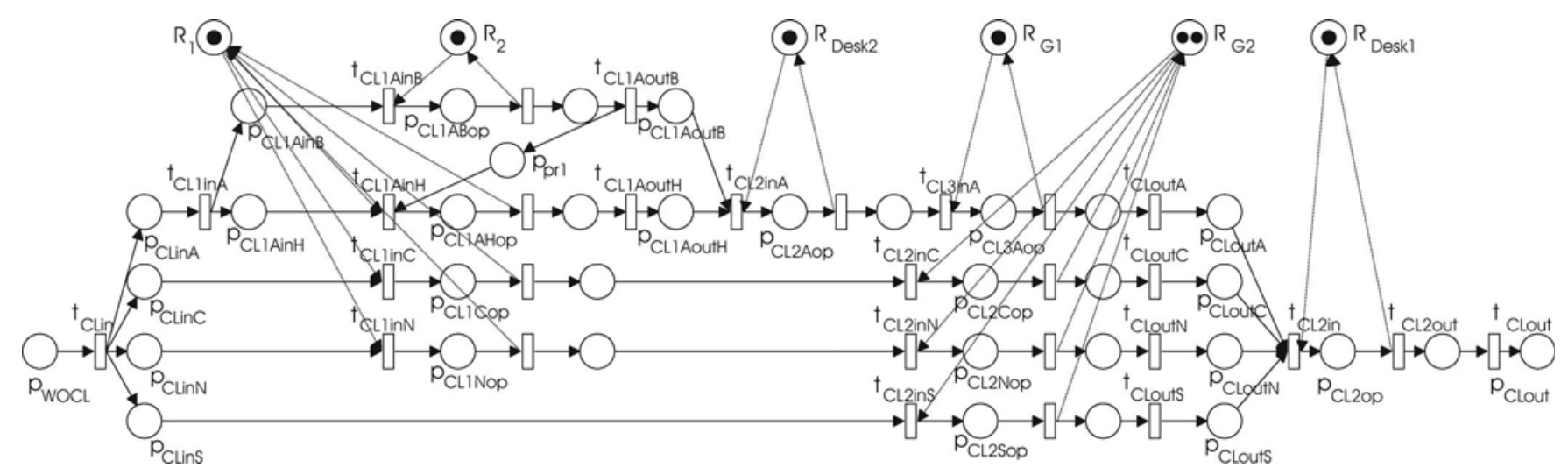

Figure 15. PN model of product 'Corner clamp L'.

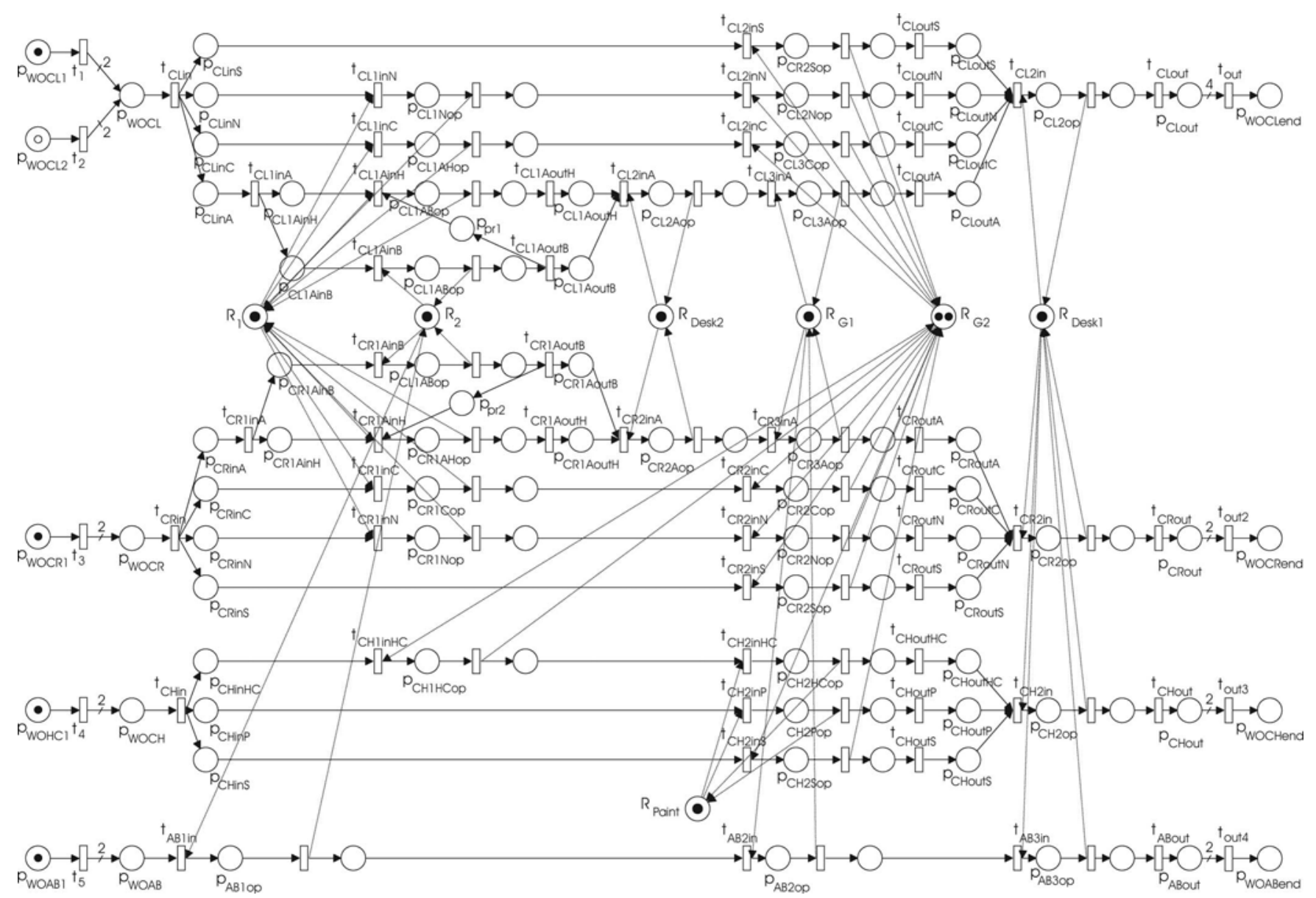

Figure 16. PN model of all products.

1996) to schedule jobs. In this way, all operations for a selected job (order) are scheduled, starting with the first operation and then forward in time to add later operations. The next job is then loaded until all the jobs have been done. The result using this tool is presented in figure 18. From this Gantt chart we can see that this schedule would finish the job in 238 time units (table 8). 

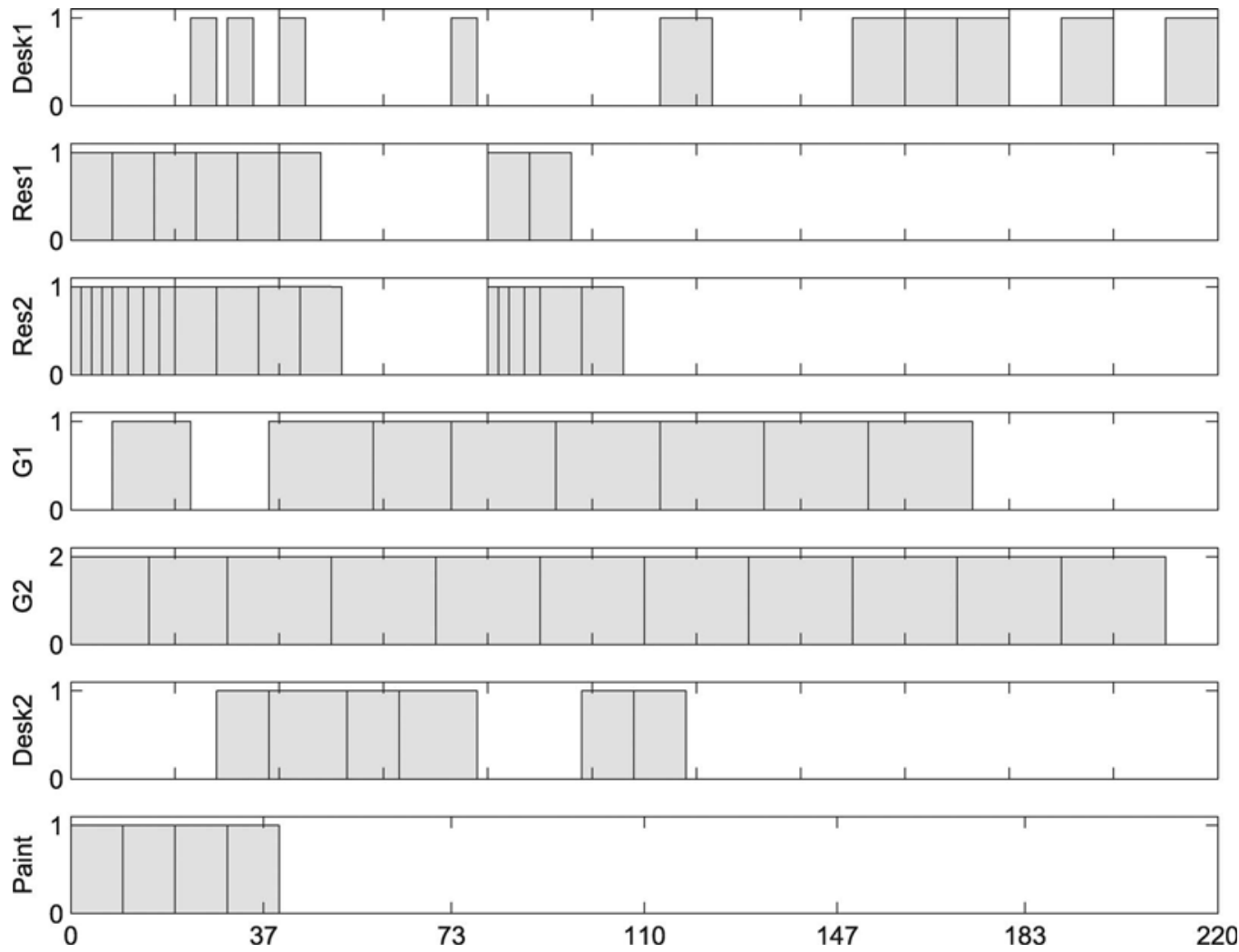

Figure 17. Schedule of the tasks in a production process using the SPT priority rule.

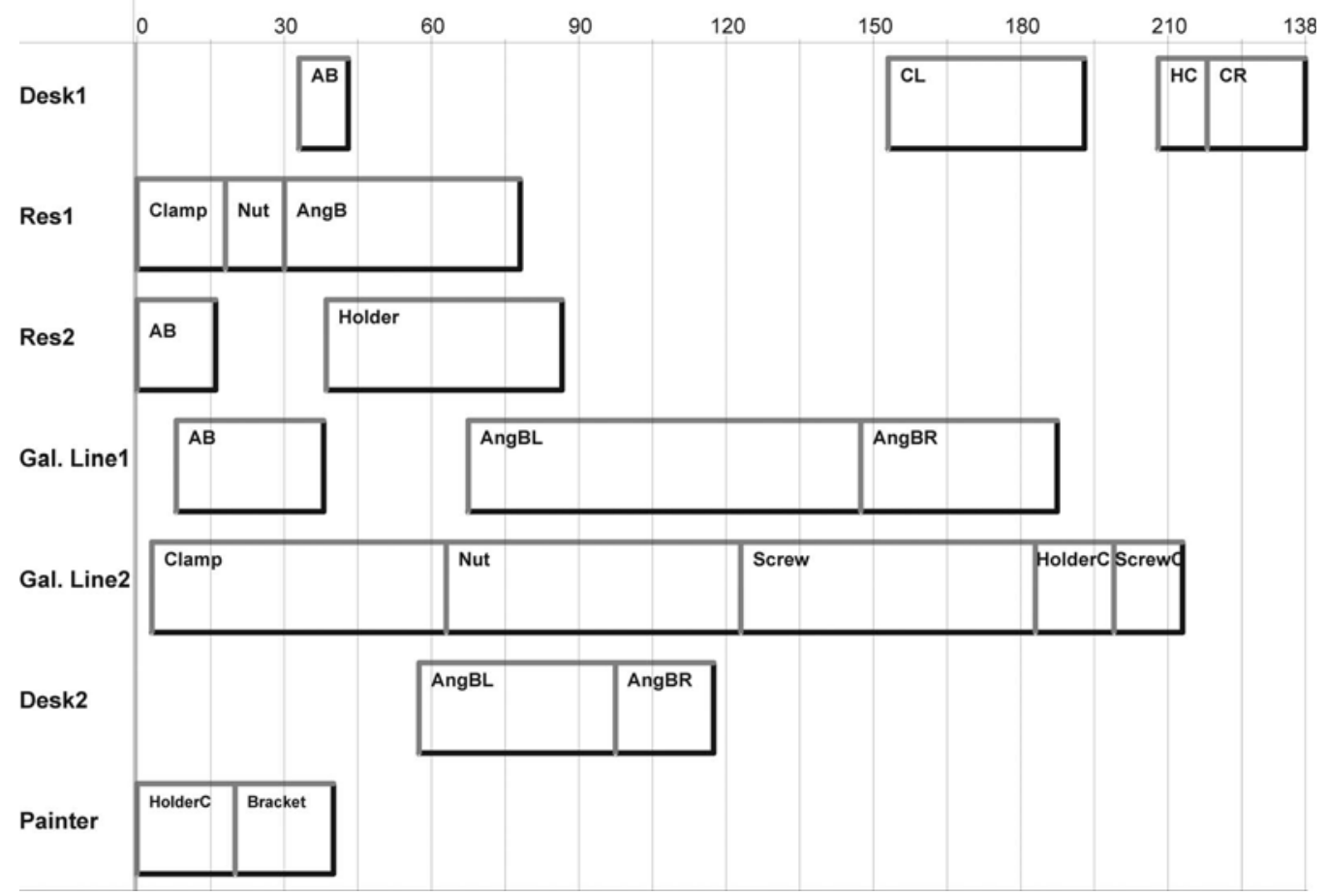

Figure 18. Schedule of the tasks in a production process using Preactor. 
Table 8. Results.

\begin{tabular}{lc}
\hline Algorithm & Time of execution \\
\hline SPT rule & 220 \\
LPT rule & 225 \\
PN-based heuristic search & 220 \\
Preactor & 238 \\
\hline
\end{tabular}

\section{Conclusion}

To be able to analyse a production system, a mathematical model of the system is required. Timed Petri nets represent a powerful mathematical formalism. In our work, timed Petri nets with the holding-duration principle of time implementation were used to automate the modelling of a type of production system described by data from production-management systems. The production data are given with the BOM and the routings. The procedure for building a model using these data is presented. For the particular timed Petri net we present a simulator that can be used to simulate models built with timed Petri nets. For the purposes of scheduling, different heuristic rules can be introduced into the simulator. The same timed Petri-net model can also be used in conjunction with other Petri-net scheduling algorithms, e.g. heuristic search. The applicability of the proposed approach was illustrated for an assembly process for producing furniture fittings. The model developed with the proposed method was used to determine a schedule for production operations. The results were compared with a commercial scheduling tool. For future work we plan to investigate the applicability of highlevel Petri nets to the proposed modelling method as well as the use of the generated models for the testing of various heuristic search algorithms.

\section{References}

Abdeddaim, Y., Asarin, E. and Maler, O., Scheduling with timed automata. Theoretical Computer Science, 2006, 354, 272-300.

Arjona-Suarez, E. and Lopez-Mellado, E., Synthesis of coloured Petri nets for FMS task specification. International Journal of Robotics and Automation, 1996, 11, 111-117.

Basile, F., Chiacchio, P., Mazzocca, N. and Vittorini, V., Modeling and control specification of flexible manufacturing systems using behavioral traces and Petri nets building blocks. Journal of Intelligent Manufacturing, to appear.

Blackstone, J.H., Phillips, D.T. and Hogg, G.L., A state-of-the-art survey of dispatching rules for manufacturing job shop operations. International Journal of Production Research, 1982, 20, 27-45.

Błażewicz, J., Domschke, W. and Pesch, E., The job shop scheduling problem: conventional and new solution techniques. European Journal of Operational Research, 1996, 93, 1-33.

Błażewicz, J., Pesch, E. and Sterna, M., The disjunctive graph machine representation of the job shop scheduling problem. European Journal of Operational Research, 2000, 127, 317-331.
Bowden, F.D.J., A brief survey and synthesis of the roles of time in Petri nets. Mathematical \& Computer Modelling, 2000, 31, 55-68.

Camurri, A., Franchi, P., Gandolfo, F. and Zaccaria, R., Petri net based process scheduling: a model of the control system of flexible manufacturing systems. Journal of Intelligent and Robotic Systems, 1993, 8, 99-123.

Czerwinski, C.S. and Luh, P.B., Scheduling products with bills of materials using an improved Lagrangian relaxation technique. IEEE Transactions on Robotics and Automation, 1994, 10, 99-111.

Enns, S.T., Finite capacity scheduling systems: performance issues and comparisons. Computers and Industrial Engineering, 1996, 30, $727-739$

Ezpeleta, J. and Colom, J.M., Automatic synthesis of colored Petri nets for the control of FMS. IEEE Transactions on Robotics and Automation, 1997, 13, 327-337.

Gu, T. and Bahri, P.A., A survey of Petri net applications in batch processes. Computers in Industry, 2002, 47, 99-111.

Hauptman, B. and Jovan, V., An approach to process producion reactive scheduling. ISA Transactions, 2004, 43, 305-318.

Huang, H.H., Lewis, F.L., Pastravanu, O.C. and Gürel, A., Flow shop scheduling design in an FMS matrix framework. Control Engineering Practice, 1995, 3, 561-568.

Jain, A.S. and Meeran, S., Deterministic job-shop scheduling: past, present and future. European Journal of Operational Research, 1999, 113, 390-434.

Janneck, J.W. and Esser, R., Higher-order petri net modelling - techniques and applications, in Formal Methods in Software Engineering and Defence Systems. Conferences on Research and Practice in Information Technology, 2002, pp. 17-25.

Jeng, M.D., A Petri net synthesis theory for modelling flexible manufacturing systems. IEEE Transactions on Systems, Man, and Cybernetics - Part B: Cybernetics, 1997, 27, 169-183.

Lee, D.Y. and DiCesare, F., Scheduling flexible manufacturing systems using Petri nets and heuristic search. IEEE Transactions on Robotics and Automation, 1994, 10, 123-132.

López-Mellado, E., Analysis of discrete event systems by simulation of timed Petri net models. Mathematics and Computers in Simulation, 2002, 61, 53-59.

Matcovschi, M.H., Mahuela, C. and Pastravanu, O., Petri net toolbox for MATLAB, in 11th IEEE Mediterranean Conference on Control and Automation, MED'03, 2003.

Murata, T., Properties, analysis and applications. Proceedings of the IEEE, 1989, 77, 541-580.

Panwalkar, S.S. and Iskaneder, W., A survey of Scheduling Rules. Operations research, 1977, 25, 45-61.

Proth, J.M., Wang, L. and Xie, X., A class of Petri nets for manufacturing system integration. IEEE Transactions on Robotics and Automation, 1997, 13, 317-326.

Ratzer, A., Wells, L., Lassen, H., Laursen, M., Qvortrup, J., Stissing, M., Westergaard, M., Christensen, S. and Jensen, K., CPN tools for editing, simulating, and analysing coloured Petri nets, in 24th ICATPN, 2003, pp. $450-462$.

Recalde, L., Silva, M., Ezpeleta, J. and Teruel, E. In Petri Nets and Manufacturing Systems: An Examples-Driven Tour, Lectures on Concurrency and Petri Nets, Vol. 3098, pp. 742-788, 2004 (Springer: Berlin).

Richard, P. and Proust, C., Solving scheduling problems using Petri nets and constraint logic programming. RAIRO - Recherche OperationnelleOperations Research, 1998, 32, 125-143.

Silva, M. and Teruel, E., Petri nets for the design and operation of manufacturing systems. European Journal of Control, 1997, 3, 82199.

Van der Aalst, W.M.P., Petri net based scheduling. OR Spectrum, 1996, 18, 219-229. 
Van der Aalst, W.M.P., The application of Petri nets to workflow management. Journal of Circuits, Systems and Computers, 1998, 8, $21-66$.

Wortmann, H., Comparison of information systems for engineer-to-order and make-to-stock situations. Computers in Industry, 1995, 26, 261271.

Xue, Y., Kieckhafer, R.M. and Choobineh, F.F., Automated construction of GSPN models for flexible manufacturing systems. Computers in Industry, 1998, 37, 17-25.

Yeh, C.H., Schedule based production. International Journal of Production Economics, 1997, 51, 235-242.

Yu, H., Reyes, A., Cang, S. and Lloyd, S., Combined Petri net modelling and AI-based heuristic hybrid search for flexible manufacturing systems Part I: Petri net modelling and heuristic search. Computers and Industrial Engineering, 2003, 44, 527-543.
Yu, H., Reyes, A., Cang, S. and Lloyd, S., Combined Petri net modelling and AI-based heuristic hybrid search for flexible manufacturing systems-Part II: Heuristic hybrid search. Computers and Industrial Engineering, 2003, 44, 545-566.

Zhou, M., DiCesare, F. and Desrochers, A.A., A hybrid methodology for synthesis of Petri net models for manufacturing systems. IEEE Transactions on Robotics and Automation, 1992, 8, 350-361.

Zhou, M.C. and Venkatesh, K., Modelling, Simulation, and Control of Flexible Manufacturing Systems: A Petri Net Approach, 1999 (World Scientific: New York).

Zuberek, W.M., Timed Petri nets: definitions, properties and applications. Microelectronics and Reliability, 1991, 31, 627-644.

Zuberek, W.M. and Kubiak, W., Timed Petri nets in modeling and analysis of simple schedules for manufacturing cells. Computers and Mathematics with Applications, 1999, 37, 191-206. 